\title{
La asociación obesidad-enfermedad psiquiátrica: una necesidad más de abordaje multidisciplinar
}

\author{
The association obesity-psychiatric disease. One more need \\ for a multidisciplinary approach
}

\author{
J. Salvador, G. Frühbeck
}

La relación entre obesidad y el desarrollo de enfermedades psiquiátricas, y especialmente depresión, ha sido objeto frecuente de atención en la literatura médica como consecuencia de la elevada prevalencia de ambas condiciones en la población y sus efectos deletéreos sobre el riesgo cardiovascular y la calidad de vida ${ }^{1-3}$. Adicionalmente, las alteraciones mentales pueden desempeñar un papel significativo en la identificación de factores etiopatogénicos implicados en el origen de la obesidad. Por tanto, establecer las posibles interrelaciones entre obesidad y patología psiquiátrica constituye un objetivo de alto interés y potencial repercusión significativa sobre la práctica clínica habitual, y por ende sobre la salud pública.

El número actual recoge una oportuna revisión ${ }^{4}$ dirigida a comentar los aspectos más relevantes de la psicopatología que acompaña a la obesidad. En general, el análisis de los estudios evaluados revela que los pacientes obesos presentan una tasa más elevada de alteraciones psiquiátricas como depresión, ansiedad, baja autoestima, fobias, personalidad antisocial y estrés que las personas sin desviación ponderal. No obstante, las discrepancias puestas de manifiesto en algunos estudios que no muestran tal asociación, establece un cierto grado de controversia, probablemente relacionado con aspectos metodológicos. Además de las dificultades que existen para adoptar una actitud diagnóstica uniforme en las grandes series publicadas, el establecimiento de la condición biológica de obesidad conlleva igualmente aspectos discutibles. En la práctica totalidad de estudios publicados tanto en relación con la psicopatología como con cualquier otro objeto de investigación que rodea a la obesidad, se hace uso del valor del índice de masa corporal (IMC) para otorgar la calificación de obesidad o sobrepeso. Sin embargo, el concepto de obesidad se define como un excesivo acúmulo de tejido adiposo, circunstancia que no se expresa fielmente con la relación existente entre peso y talla. En este sentido, un estudio

Departamento de Endocrinología y Nutrición. Clínica Universidad de Navarra.

\author{
Correspondencia \\ Javier Salvador \\ Departamento de Endocrinología y Nutrición \\ Clínica Universidad de Navarra \\ Avda Pío XII, 36 \\ 31008 Pamplona \\ E-mail.jsalvador@unav.es
}


recientemente publicado basado en la comparación entre la cifra de IMC y la valoración de la composición corporal pone de manifiesto que un elevado porcentaje de pacientes con IMC inferior a $30 \mathrm{~kg} / \mathrm{m}^{2}$ presentan un aumento del compartimento graso compatible con obesidad ${ }^{5}$. Por tanto, si empleamos la medida de adiposidad como parámetro biológico y conceptualmente válido encontramos que la prevalencia de obesidad es mucho más elevada que lo estimado mediante IMC, y que pacientes caracterizados como normoponderales o con sobrepeso son en realidad obesos, artefactando los resultados que establecen la relación entre obesidad y alteración psicopatológica. Por otra parte, es la obesidad visceral el factor que se encuentra especialmente implicado en la fisiopatología de la mayoría de comorbilidades derivadas de la obesidad, y evaluar este parámetro mediante medidas indirectas como el perímetro de cintura no siempre ofrece una fiabilidad y reproducibilidad suficiente, especialmente en pacientes con obesidad mórbida ${ }^{1-3}$. En otro orden de cosas, la etiología multifactorial tanto de las enfermedades psiquiátricas como de la obesidad complica la búsqueda de una relación lineal entre la prevalencia o el desarrollo de ambas condiciones.

Sin embargo, a pesar de estas limitaciones, investigaciones recientes derivadas de los datos recogidos en el Nurses Health Study ${ }^{6}$ concluyen en la existencia de una relación bidireccional entre obesidad y depresión, sugiriendo que las mujeres con obesidad muestran mayor probabilidad de desarrollo de depresión y que aquellas con depresión muestran un riesgo aumentado de desarrollo de obesidad después de ajustar los datos por variables como edad, actividad física y comorbilidades asociadas. Otra evaluación de un número limitado de estudios orientados a explorar la relación obesidad-depresión revela que se advierte asociación entre obesidad y depresión en el $80 \%$ de las publicaciones diseñadas con tal fin, mientras que la aparición de obesidad en pacientes con depresión se constató en el 53\% de los estudios, sugiriendo igualmente una interacción bilateral entre ambas condiciones clínicas $^{7}$. Otro metanálisis reciente concluye en un efecto potenciador de la obesidad y del sobrepeso sobre el riesgo evolutivo de desarrollar depresión, así como de la depresión para predecir la obesidad a mediolargo plazo $^{8}$, insistiendo en la bidireccionalidad de la influencia de origen y desarrollo entre ambas enfermedades. Los efectos beneficiosos de la intervención sobre los síntomas depresivos del estilo de vida en un metanálisis que incluye 31 estudios sustenta la participación de elementos relacionados con el tratamiento de la obesidad, aunque no se demostraron relaciones con los cambios ponderales. Por otra parte, la mejoría en los síntomas depresivos se asocia a evolución favorable del tratamiento de la obesidad, al menos durante los primeros seis meses de intervención terapéutica, lo que junto al curso satisfactorio de los parámetros psicológicos que acompaña a la reducción ponderal asociada a la cirugía bariátrica, proporcionan nuevos soportes a la asociación entre ambas patologías 9 .

Las posibilidades etiopatogénicas, que potencialmente se ven implicadas en estas relaciones, son variadas. La observación de una mayor tasa de enfermedades nutricionales, metabólicas y cardiovasculares en pacientes con enfermedad mental severa aboga por una participación de factores generales que pueden agruparse alrededor de este tipo de 
patología, como alimentación inadecuada, sedentarismo, tabaquismo y otros estilos de vida y cuidados poco saludables ${ }^{10}$. La frecuente asociación de trastornos del sueño y su sugerida implicación con el desarrollo de un perfil hormonal estimulador de la ingesta (aumento de ghrelina y reducción de leptina) puede ejercer un papel contributorio significativo ${ }^{11}$. La elevada prevalencia de síndrome de apneas obstructivas del sueño en pacientes con obesidad mórbida y su asociación con alteraciones psicopatológicas, insomnio y reducción de calidad de vida representa un mecanismo adicional que colabora a vincular obesidad y psicopatología ${ }^{12}$. La reacción de estrés que provoca una hiperactividad del sistema nervioso simpático y del eje hipotálamo-hipófiso-adrenal, y aumenta la producción de catecolaminas y cortisol es otro candidato a explicar por qué los pacientes con enfermedad psiquiátrica severa poseen mayor frecuencia de obesidad visceral y riesgo cardiovascular más elevado ${ }^{13}$. En otro orden, existen datos experimentales que sugieren que la resistencia a la leptina, prácticamente universal en la obesidad, contribuye al desarrollo de depresión ${ }^{14}$. En cualquier caso, algunas enfermedades psiquiátricas, como la depresión mayor, favorecen la potenciación del patrón proinflamatorio propio de la obesidad que contribuye a incrementar la resistencia insulínica y el riesgo de diabetes, dislipemia e hipertensión ${ }^{15}$. Mención adicional merecen los efectos de fármacos antipsicóticos sobre el desarrollo de obesidad, diabetes y síndrome metabólico, cuyo manejo requiere de la colaboración coordinada de diferentes especialistas ${ }^{16}$.

Por consiguiente, los datos epidemiológicos y fisiopatológicos disponibles ofrecen soporte a la relación bilateral entre obesidad y psicopatología y proporcionan una ventana terapéutica para optimizar el tratamiento de ambas condiciones. La consecuencia inmediata es la necesidad del trabajo multidisciplinar consensuado entre psiquiatras, endocrinólogos, internistas y médicos de atención primaria tanto en la vertiente asistencial como investigadora que permita optimizar el tratamiento de enfermedades tan prevalentes y con un alto impacto en la calidad de vida de la población y en el gasto sanitario.

\section{BIBLIOGRAFÍA}

1. Salvador J, Silva C, Pujante P, Frühbeck G. Obesidad abdominal: un estandarte del riesgo cardiometabólico. Endocrinol Nutr 2008; 55: 420-432.

2. Dixon JB. The effect of obesity on health outcomes. Mol Cell Endocrinol 2010; 316: 104-108.

3. Yusuf S, Hawken S, Ounpuu S, Dans T, Avezum A, Lanas F et al. Effect of potentially modifiable risk factors associated with myocardial infarction in 52 countries (the INTERHEART study): case-control study. Lancet 2004; 364: 937-952.

4. Baile JI, Gonzalez MJ. Comorbilidad psicopatológica en obesidad. An Sist Sanit Navar 2011: 34; 253-261.

5. Gómez-Ambrosi J, Silva C, Galofré JC, Escalada J, Santos S, Millán D et al. Body mass index classification misses subjects with increased cardiometabolic risk factors related to elevated adiposity. Int J Obes (Lond) 2011 May 17. [Epubahead of print] PubMed PMID: 21587201.

6. Pan A, Sun Q, CZernichow S, Kivimaki, OKereke OI, Lucas M et al. Bidirectional association between depression and obesity in middle-aged and older women. Int J Obes (Lond) 2011; Jun 7 doi:10.1038/ijo.2011.111 [Epub ahead of print]. 
7. Faith MS, Butryn M, Wadden TA, Fabricatore A, Nguyen AM, Heymsfield SB. Evidence for prospective associations among depression and obesity in population-based studies. Obes Rev 2011 May 12(5):e438-53.doi:10.1111/j.1467789X.2010.00843x.

8. Luppino FS, de Wit LM, Bouvy PF, Stijnen T, CujJpers P, Pennimx BWJH et al. Overweight, obesity and depression. Arch Gen Psychiatry 2010; 67: 220-229.

9. Simon GE, Rohde P, Ludman EJ, JefFery RW, Linde JA, Operskalski BH et al. Association between change in depression and change in weight among women enrolled in weight loss treatment. Gen Hosp Psychiatry 2010; 32: 583-589.

10. De Hert M, Correll CU, Bobes J, Cetkovich-Bakmas M, Cohen D, Asai I et al. Physycal illness in patients with severe mental disorders. I. Prevalence, impact of medications and disparities in health care. World Psychiatry 2011; 10: 52-57.

11. Spiegel K, Tasali E, Penev P, Van Cauter E. Brief communication: Sleep curtailment in healthy young men is associated with decreased leptin levels, elevated ghrelin levels, and increased hunger and appetite. Ann Intern Med 2004; 141: 846-850.

12. Macey PM, Woo MA, Kumar R, Cross RL, Harper R. Relationship between obstructive sleep apnea severity and sleep, depression and anxiety symptoms in newly-diagnosed patients. PLoS One 2010; 5(4): e10211.

13. Chrousos GP. Stress and disorders of the stress system. Nat Rev Endocrinol 2009; 5: 374-381.

14. Yamada N, Katsuura G, Ochi Y, Ebihara K, Kusakabe T, Hosoda K et al. Impaired CNS leptin action is implicated in depression associated with obesity. Endocrinology 2011 ; doi:10.1210/en.2011-0004.

15. Howren MB, LAMKIn DM, Suls J. Association of depression wioth C-reactive protein, IL-1, and IL-6: a meta-analysis. Psychosom Med 2009; 71: 171-186.

16. Rodríguez Artalejo F, Baca E, Esmatjes E, Merino JF, Monereo S, Moreno B et al. Valoración y control del riesgo metabólico y cardiovascular en los pacientes con esquizofrenia. Med Clin (Barc) 2006; 127: 542-548. 